\title{
Morphological plasticity of ticks' salivary glands and the meaning of hematophagy in hosts immunized with glandular extract of females fed for 4 days
}

\author{
Karim Christina Scopinho Furquim ${ }^{1,}$, , Maria Izabel Camargo Mathias ${ }^{1}$, Gislaine Cristina Roma ${ }^{1}$, \\ Letícia Maria Gráballos Ferraz Hebling ${ }^{1}$, Gervásio Henrique Bechara ${ }^{2}$ \\ ${ }^{1}$ Departamento de Biologia, Instituto de Biociências, UNESP, Av. 24 A, nº 1515, Cx. Postal 199, CEP: 13506-900, Rio Claro, S.P., Brazil \\ ${ }^{2}$ Departamento de Patologia Veterinária, FCAV, UNESP, Via de Acesso Prof. Paulo Castellane, s/n, CEP: 14884-900, Jaboticabal, S.P., Brazil
}

Email address:

karimfurquim@yahoo.com.br (K. C. S. Furquim)

To cite this article:

Karim Christina Scopinho Furquim, Maria Izabel Camargo Mathias, Gislaine Cristina Roma, Letícia Maria Gráballos Ferraz Hebling, Gervásio Henrique Bechara. Morphological Plasticity of Ticks' Salivary Glands and the Meaning of Hematophagy in Hosts Immunized with Glandular Extract of Females Fed for 4 Days. Animal and Veterinary Sciences. Vol. 2, No. 6, 2014, pp. 194-207.

doi: 10.11648/j.avs.20140206.17

\begin{abstract}
A histochemical analysis was performed in this study in order to detect proteins, polysaccharides, lipids, acid phosphatase and calcium in the secretion produced by the salivary glands of Rhipicephalus sanguineus females (Latreille, 1806) (Acari, Ixodidae) fed for 2, 4 and 6 days in New Zealand White rabbits which had been previously immunized with glandular extract obtained from females fed for 4 days (SGE4). The results revealed that such glands presented alterations in their secretory cycle, which occurred: a) by the inactivity of some $c 1$ cells (in the glands of females fed for 2 days) and $c 4$ (in those fed for 4 days) and b) by the modification in the constitution of the secretion of females fed for 2-6 days. It was verified that, in the glands of females fed for 2 days, there was an increase in proteins and calcium; a reduction in lipids and the contents of polysaccharides and acid phosphatase remained unaltered. In those fed for 4 days there was an increase in proteins, calcium and acid phosphatase; reduction in the lipids, and the content of polysaccharides remained unaltered. In the females fed for 6 days an increase in the components was observed; however, there was a reduction in lipids and acid phosphatase. In addition, it was verified that, in a decrescent order of histochemical alterations, the most affected cells were: $f ; c 2, c 3, c 5 ; a, d$ and $c 4, e$ in the glands of females fed for 2 days; $c 5 ; a, c 2, c 3, d$ and $c 1, e$ in the glands of females fed for 4 days and $a, c 1, c 2, d$ and $e, c 3$ in the glands of females fed for 6 days. The data here obtained clearly show that the most pronounced histochemical modifications were detected in the glands of females fed for 2 and 6 days; however, the modifications observed in the females fed for 4 days must also be considered, once they were significant as well. This has probably occurred because the hosts were inoculated with SGE4, obtained from the salivary glands of females fed for 4 days, intermediate stage of the glandular cycle, which contains antigens that are common to glandular tissues of females fed for 2 and 6 days.
\end{abstract}

Keywords: Rhipicephalus sanguineus, Immunization, Antigen, Salivary Gland, Secretory Cycle, Histochemistry, Immune-Inflammatory Response

\section{Introduction}

Over their evolutionary process the ticks left their condition of saprophage and became hematophage, due to the adaptation of their behavior, anatomy and the activity of their salivary glands [1]. The latter allowed these ectoparasites to develop a sophisticated mechanism of secretion, able to overcome the hemostatic and immunologic barriers of their hosts [2].
The salivary glands then started to present a morphofunctional complexity. In Ixodidae females, they comprise three types of acini: I, II and III [3-6].

Acini II and III are glandular; i.e., they have secretory function; therefore, they are considered the most important for the parasite-host relationship [7]. Acini II are formed by $a$, $b, c 1, c 2, c 3$ and $c 4$ secretory cells [3]. In addition to these, 
$c 5$ and $c 6$ cells have recently been described in $R$. sanguineus females [8]. Acini III comprise $d, e$ and $f$ cells [3-5, 8].

The ticks' saliva contains bioactive molecules of glycoprotein, lipoprotein and lipidic nature, such as acid phosphatase, esterases, aminopeptidases, metaloproteases, calreticulin, prostaglandins, lipocalins and others, which vary qualitatively and quantitatively depending on the species [9].

In this sense, the study of the salivary glands of Boophilus microplus females demonstrated the production of: a) glycoproteins by $b, c 1, c 2, c 3$ cells of acinus II and $f$ of III; b) acid phosphatase by three types of acini, mainly by I and by $d$ cells of acinus III; c) proteases by cells of acinus II; d) esterases by cells of all the acini, mainly $b$ and $c l$ cells of acinus II and e) lipoproteins by $a$ cells of acinus II and $d$ and $e$ cells of acinus III [3].

According to literature, the ticks' saliva is considered a heterogeneous mixture, extremely complex and efficient, once it is able to overcome the barriers offered by the host very quickly, on the first days of the feeding process $[1,10$ 12]. On the other hand, the saliva also contains antigens which are able to activate the host's immune response [1], which changes the interface of the relationship parasite-host [9], interfering on the tick's blood feeding [13]. According to reference [14], the antigenic determinants in these glandular antigens are not only the proteins, but also the polysaccharides, an interesting fact, once most numerous components of the saliva are the glycoproteins.

Thus, this study had the objective to histochemically investigate the secretory cycle of the salivary glands of $R$. sanguineus females fed for 2, 4 and 6 days in rabbits previously immunized with extracts of salivary glands of females from this species fed for 4 days.

\section{Material and Methods}

\subsection{Material}

To perform this study salivary glands of adult $R$. sanguineus females fed for 2, 4 and 6 days subjected to infestation on New Zealand White rabbits immunized with glandular extract of females fed for 4 days were used. For this, unfed individuals (males and females) from a colony kept in BOD incubator, in controlled conditions $\left(29^{\circ} \mathrm{C}, 80 \%\right.$ of humidity and photoperiod of 12 hours), in the Biotery of the Biology Department of UNESP campus Rio Claro (SP), Brazil were used in the infestations A, B e C made in rabbits according to the procedure described by the study [15].

- Infestation A: made in naive rabbits using 25 couples of $R$. sanguineus couples/host, for the acquisition of females of ticks fed for 4 days (36 individuals), for the obtainance of glandular extract: SGE4= glandular extract of females fed for 4 days.

The extract was processed and inoculated in the hosts subjected to infestation B.

- Infestation B (test group = TG): made in 4 rabbits sensibilized with SGE4 extract, which were subjected to challenge infestation with 15 couples of adult $R$. sanguineus ticks/host.

- Infestation $C$ (control group= $C G$ ): made in 4 naive rabbits that were not inoculated, which were subjected to challenge infestation with 15 couples of adult $R$. sanguineus ticks/host.

This experiment was approved by the Ethics Committee in Research and Scientific Merit- UNIARARAS, Protocol $n^{\circ}$ 021/2009.

\subsection{Methods}

The SGE4 extract was prepared in the Molecular Biology Laboratory of the Biology Department, UNESP campus Rio Claro (SP), Brazil, and the host immunization was realized in the Biotery of the Biology Department, UNESP campus Rio Claro (SP). The procedures for obtaining of the extract and hosts immunization occurred according to literature procedure [16].

The challenge infestation was realized after 15 days from the last inoculation. All the hosts from TG and CG groups were subjected to with 15 couples of $R$. sanguineus/host. Then, $R$. sanguineus females fed for 2, 4 and 6 days were removed from the rabbits inoculated with SGE4 extract (GT), as well as from those not inoculated (CG) and subjected to histochemistry analysis.

In the Histology Laboratory of the Biology Department of UNESP campus Rio Claro (SP), Brazil, salivary glands of $R$. sanguineus females of each group (TG and CG) fed for 2, 4 and 6 days were removed in buffered saline solution $(7.5 \mathrm{~g}$ $\mathrm{NaCl}+2.38 \mathrm{~g} \mathrm{Na}_{2} \mathrm{HPO}_{4}+2.72 \mathrm{~g} \mathrm{KH}_{2} \mathrm{PO}_{4}$ in $1000 \mathrm{~mL}$ distilled water) and fixed.

Part of the material was fixed in $4 \%$ paraformaldehyde at $4^{\circ} \mathrm{C}$, after fixation, the material was dehydrated in a series of increasing concentrations of ethanol $(70 \%, 80 \%, 90 \%$ and $95 \%$ ), embedded in resin (Leica), and sectioned at $3 \mu \mathrm{m}$ thickness. Sections were mounted on glass slides and histochemical tests were applied to detect the presence of the following compounds: proteins (bromophenol blue) [17]; polysaccharides (PAS- Periodic Acid Schiff) [18]; calcium (von Kossa) [19] and lipids [20].

The other part of the material was fixed in $10 \%$ buffered neutral formalin and acetone (9:1) for one hour and thirty minutes at $4^{\circ} \mathrm{C}$, then processed according to the methods described by this reference [21] for detection of acid phosphatase activity. The material was then dehydrated in increasing concentrations of ethanol $(70 \%, 80 \%, 90 \%$ and $95 \%$ ), embedded in resin (Leica), and sectioned at a thickness of $7 \mu \mathrm{m}$. The sections were placed on glass slides and counterstained with hematoxylin for 2 minutes. The control samples were incubated without substrate.

The slides of the all histochemical tests were mounted with Canada balsam and examined and photographed under Motic BA 300 light microscope.

\section{Results}

The histochemical results for the salivary glands of R.sanguineus females fed for 2, 4 and 6 days from the 
Control Group (CG) were presented in a previously study [16].

\subsection{Females of the Test Group Fed for 2 Days}

Acinus II

- a cells: the secretion granules are moderately (Figs. 1A, $3 \mathrm{~A})$ or strongly (Figs. 1B-D, 3B) stained for bromophenol blue and Baker techniques; negative (Figs. 2A, D-E) or weakly (Figs. 2B-C) stained for PAS, and negative for acid phosphatase (Figs. 4A-C, E) and von Kossa (Figs. 5A-C).

- c1cells: are not observed for being inactive in this phase.

- c2 cells: the granules are weakly positive for bromophenol blue technique (Fig. 1B); moderately stained for PAS (Figs. 2A-C) and Baker (Fig. 3A); weakly (Fig. 4A) or moderately (Fig. 4B) positive for acid phosphatase and negative for von Kossa (Figs. 5AB).

- c3 cells: granules are strongly stained for bromophenol blue (Fig. 1C), PAS (Figs. 2A, C) and von Kossa (Figs. 5B-C) techniques and moderately stained for Baker (Fig. 3C) and acid phosphatase (Fig. 4C).

- c4 cells: granules are negative for all the applied techniques (Figs. 1D, 2A, 3A, 4D, 5A).

- c5 cells: the granules are weakly stained for bromophenol blue (Figs. 1A-B); moderately stained for acid phosphatase (Figs. 4D-E) and negative for von Kossa (Fig. 5C). As for PAS and Baker, either moderately (Figs. 2C, 3D) or strongly (Figs. 2E, 3E) stained granules or heterogeneous staining (moderate in one region and strong in other) (Figs. 2D, 3F) is observed.

Acinus III

- d cells: the granules are strongly positive for bromophenol blue (Fig. 1E); negative for PAS (Figs. 2F-H) and von Kossa (Fig. 5D); weakly (Fig. 3G) or strongly (Fig. 3H) stained for Baker and negative (Fig. $4 \mathrm{~F}$ ) or present heterogeneous (negative and weak) for acid phosphatase (Figs. 4G-H).

- e cells: the granules are weakly (Fig. 1E) or moderately (Figs. 1E-F) stained for bromophenol blue; negative for PAS (Figs. 2F-H), acid phosphatase (Figs. 4F-H) and von Kossa (Figs. 5D-E) and weakly stained for Baker (Figs. 3G-I).

- f cells: the granules are weakly (Fig. 1E) or moderately (Figs. E-F) stained for bromophenol blue; moderately (Fig. 3H) or strongly (Fig. 3I) stained for Baker; weakly (Fig. 4G), moderately (Fig. 4F) or strongly (Fig. 4H) stained for acid phosphatase and strongly positive for von Kossa (Fig. 5E). For PAS, the granules are weakly stained (Fig. $2 \mathrm{H}$ ) or present heterogeneous staining; i.e., weak in one region and moderate in other (Fig. 2G), or even moderate in one region and strong in other (Fig. 2F).

\subsection{Females of the Test Group Fed for 4 Days}

Acinus II

- $a$ cells: the granules are moderately (Figs. 1G-I) stained for bromophenol blue and Baker (Fig. 3J) techniques; negative for PAS (Figs. 2I-L), acid phosphatase (Figs. 4I-M) and von Kossa (Figs. 5F, H).

- clcells: the granules are moderately stained for bromophenol blue (Fig. 1G), strongly stained for PAS (Fig. 2I) and von Kossa (Fig. 5G) and weakly (Fig. 4I) or moderately (Fig. 4J) stained for acid phosphatase. These cells are not observed through the application of Baker technique.

- $c 2$ cells: the granules are weakly stained for bromophenol blue (Figs. 1G-H) and Baker (Fig. 3J); weakly (Figs. 2I, K-L, 4I) or moderately (Figs. 2J, 4K) stained for PAS and acid phosphatase, and negative for von Kossa (Fig. 5F).

- $c 3$ cells: the granules are moderately stained for bromophenol blue (Fig. 1H); strongly stained for PAS (Fig. 2K) and von Kossa (Fig. 5H), and weakly stained for acid phosphatase (Fig. 4L). These cells are not observed through the application of Baker technique.

- $c 4$ cells: are not observed for being inactive in this phase.

- $c 5$ cells: the granules are weakly stained for bromophenol blue (Fig. 1I); moderately stained for PAS (Fig. 2L); strongly stained for Baker (Fig. 3J); weakly stained (Figs. 4J-K) or presenting heterogeneous staining (weak in one region and moderate in other) (Fig. 4M) for acid phosphatase and negative for von Kossa (Fig. 5F).

Acinus III

- $d$ cells: the granules are moderately (Fig. 1J) or strongly (Fig. 1K) stained for bromophenol blue; negative for PAS (Fig. 2M) and von Kossa (Fig. 5I); strongly stained for Baker (Figs. 3K-L), and presenting heterogeneous staining (negative in ne region and weak in other) for acid phosphatase(Fig. 4N).

- $e$ cells: the granules are weakly stained for bromophenol blue (Figs. 1J-K); negative for PAS (Fig. 2M), acid phosphatase (Fig. 4N) and von Kossa (Fig. 5I), and moderately stained for Baker (Figs. 3K-L).

- $f$ cells: in this phase of the secretory cycle these cells no longer contain secretion granules (Figs. 1J, 2M, 4N, 5I).

\subsection{Females of the test group fed for 6 days}

Acinus II

- $a$ cells: the granules are moderately (Fig. 1L) or strongly (Fig. 1M) stained for bromophenol blue; negative (Figs. 2N, P) or weakly (Fig. 2O) stained for PAS; weakly (Figs. 3M, O) or moderately (Fig. 3N) stained for Baker and negative for acid phosphatase (Fig. 4O) and von Kossa (Figs. 5J-L).

- $c 1$ cells: the granules are strongly stained for bromophenol blue (Fig. 1L), PAS (Figs. 2N-O) and von Kossa (Fig. 5K) and negative for acid phosphatase (Fig. 
4P). Such cells are not observed through the application of Baker technique.

- $c 2$ cells: the granules are weakly stained for bromophenol blue(Fig. 1M); moderately stained for Baker (Figs. 3M-N) and negative for von Kossa (Fig. 5J). For PAS both moderately stained granules (Fig. 2N) and presenting heterogeneous staining (weakly stained in one region and moderately in other) (Fig. 2P) were observed. As for acid phosphatase, heterogeneous staining was also observed (negative in one region and weakly stained in other) (Figs. 4O-P).

- $c 3$ cells: the granules are moderately stained for bromophenol blue (Fig. 1N) and Baker (Fig. 3O); strongly stained for PAS (Figs. 2N-O) and von Kossa (Figs. 5J-L) and negative for acid phosphatase (Figs. 4O, Q-R).

- $c 4$ cells: the granules are negative for Baker (Figs. 3MO), acid phosphatase (Fig. 4R) and von Kossa (Fig. 5L). Such cells are not observed through the application of bromophenol blue and PAS techniques.

- $c 5$ cells: are not observed for being inactive in this phase.
Acinus III

- $d$ cells: the granules are strongly stained for bromophenol blue (Fig. 1O) and Baker (Fig. 3P); weakly stained (Fig. 2Q) or present heterogeneous staining (negative in one region and weakly stained in other) (Fig. 2R) for PAS; present heterogeneous staining (negative in one region and weakly stained in other) for acid phosphatase (Fig. 4S), and negative for von Kossa (Figs. 5M-N).

- $e$ cells: the granules are weakly stained (Fig. 1O) or present heterogeneous staining (weak in one region and moderately stained in other) (Fig. 1P) for bromophenol blue; negative (Fig. 2Q) or weakly (Fig. 2R) stained for PAS; moderately stained for Baker (Figs. 3P-Q) and negative for acid phosphatase (Fig. 4S) and von Kossa (Figs. 5M-N).

- $f$ cells: in this phase of the secretory cycle these cells no longer contain secretory granules (Figs. 1O-P, 2Q-R, $3 \mathrm{P}-\mathrm{Q}, 4 \mathrm{~S}, 5 \mathrm{~N})$.

The results for salivary glands of the females from groups $\mathrm{CG}$ and TG fed for 2, 4 and 6 days are summarized in tables $1-8$.

Table 1. Histochemistry of the glandular acini secretion of R. sanguineus females from groups $C G$ and $T G$ fed for 2 days.

\begin{tabular}{|c|c|c|c|c|c|c|c|c|c|c|c|}
\hline \multirow{3}{*}{ Acini } & \multirow{3}{*}{ Cells } & \multicolumn{10}{|c|}{ Study Groups } \\
\hline & & \multicolumn{5}{|c|}{ CG } & \multicolumn{5}{|c|}{ TG } \\
\hline & & BB & PAS & B & AP & VK & BB & PAS & B & AP & VK \\
\hline \multirow{6}{*}{ II } & $a$ & 2; 3 & ( & 3 & 0 & 0 & 2; 3 & $\mathbf{0} ; \mathbf{0}$ & 2; 3 & o & 0 \\
\hline & $c 1$ & 3 & 3 & 3 & $\phi$ & 3 & $*$ & $*$ & $*$ & $*$ & $*$ \\
\hline & $c 2$ & ( & $\mathbf{0} ; 2$ & ( & 2 & 0 & ( & 2 & 2 & $\mathbf{0} ; 2$ & 0 \\
\hline & $c 3$ & 2 & 3 & 3 & 2 & 0; 3 & 3 & 3 & 2 & 2 & 3 \\
\hline & $c 4$ & 0 & 0 & 0 & 2 & o & 0 & 0 & 0 & 0 & 0 \\
\hline & $c 5$ & ( ; 2 & $\mathbf{0} ; 2$ & 2; 3 & 2 & 0 & ( & 2; 3 & 2; 3 & 2 & 0 \\
\hline \multirow{3}{*}{ III } & $d$ & 3 & 0 & 3 & 0 & $\mathbf{0}$ & 3 & 0 & $\mathbf{0} ; \mathbf{3}$ & 0; $\mathbf{0}$ & 0 \\
\hline & $e$ & $\mathbf{0} ; 2$ & 0 & 2 & 0 & 0 & $\mathbf{0} ; 2$ & 0 & ( & $\mathbf{0}$ & 0 \\
\hline & $f$ & $\mathbf{0} ; \mathbf{0}$ & 2; 3 & 2; 3 & ( ; 2 & $\boldsymbol{0}$ & (); 2 & (1; 2; 3 & 2; 3 & (); 2; 3 & 3 \\
\hline
\end{tabular}

The results for the salivary glands females fed for 2, 4 and 6 days from the CG were presented in a previously study [16].

BB: bromophenol; PAS: PAS; B: Baker; AP: phosphatase; VK: von Kossa; *: inactive cell; $\phi$ : not observed cell; the intensity of staining varied from 0-4, being: $\mathbf{0}$ : negative and $\mathbf{4}$ : strongly positive.

Table 2. Histochemistry of the glandular acini secretion of $R$. sanguineus females from groups $C G$ and TG fed for 4 days.

\begin{tabular}{|c|c|c|c|c|c|c|c|c|c|c|c|}
\hline \multirow{3}{*}{ Acini } & \multirow{3}{*}{ Cells } & \multicolumn{10}{|c|}{ Study Groups } \\
\hline & & \multicolumn{5}{|c|}{ CG } & \multicolumn{5}{|c|}{ TG } \\
\hline & & BB & PAS & B & $\mathbf{A P}$ & VK & BB & PAS & B & $\mathbf{A P}$ & VK \\
\hline \multirow{6}{*}{ II } & $a$ & (1; 2 & 0 & 3 & 0 & 0 & 2 & 0 & 2 & 0 & 0 \\
\hline & $c 1$ & 2 & 3 & $\phi$ & 0 & 3 & 2 & 3 & $\phi$ & $\mathbf{0} ; 2$ & 3 \\
\hline & $c 2$ & (1) & 2 & $\phi$ & 2 & 0 & ( & (1) 2 & (1) & (1) 2 & 0 \\
\hline & $c 3$ & 2 & 3 & 3 & 0 & 0; 3 & 2 & 3 & $\phi$ & (1) & 3 \\
\hline & $c 4$ & 0 & 0 & (1) & 0; $\mathbf{0}$ & 0 & $*$ & $*$ & $*$ & $*$ & $*$ \\
\hline & $c 5$ & 2 & (1; 2 & $\phi$ & 2 & 0 & (1) & 2 & 3 & (1; 2 & 0 \\
\hline \multirow{2}{*}{ III } & $d$ & 2 & 0 & 3 & 0 & 0 & 2; 3 & 0 & 3 & $\mathbf{0} ; \mathbf{1}$ & 0 \\
\hline & $e$ & (1) & 0 & 2 & 0 & 0 & (1) & 0 & 2 & 0 & 0 \\
\hline
\end{tabular}

The results for the salivary glands females fed for 2, 4 and 6 days from the CG were presented in a previously study [16].

BB: bromophenol; PAS: PAS; B: Baker; AP: phosphatase; VK: von Kossa; *: inactive cell; $\phi$ : not observed cell; the intensity of staining varied from 0-4, 
being: $\mathbf{0}$ : negative and $\mathbf{4}$ : strongly positive.

Table 3. Histochemistry of the glandular acini secretion of $R$. sanguineus females from groups $C G$ and TG fed for 6 days.

\begin{tabular}{|c|c|c|c|c|c|c|c|c|c|c|c|}
\hline \multirow{3}{*}{ Acini } & \multirow{3}{*}{ Cells } & \multicolumn{10}{|c|}{ Study Groups } \\
\hline & & \multicolumn{5}{|c|}{ CG } & \multicolumn{5}{|c|}{ TG } \\
\hline & & BB & PAS & B & AP & VK & BB & PAS & B & AP & VK \\
\hline \multirow{6}{*}{ II } & $a$ & $\mathbf{0} ; 0$ & 0 & 3 & o & 0 & (2); & $\mathbf{0} ; \mathbf{0}$ & $\mathbf{0} ; 0$ & 0 & 0 \\
\hline & $c 1$ & (2) & 3 & 3 & 2 & 0 & (3) & B & $\phi$ & o & 3 \\
\hline & $c 2$ & - & 2 & 2 & - & 0 & - & $\mathbf{0} ; 0$ & $\mathbf{0} ; 0$ & $\mathbf{0} ; \mathbf{0}$ & o \\
\hline & $c 3$ & (2) & 3 & 3 & 0 & 2 & 2 & 3 & 2 & 0 & 3 \\
\hline & $c 4$ & 0 & 0 & 0 & 0 & 0 & $\phi$ & $\phi$ & 0 & 0 & o \\
\hline & $c 5$ & * & * & * & * & * & * & * & * & * & * \\
\hline \multirow{2}{*}{ III } & $d$ & 0; 3 & o & B & o & 0 & B & $\mathbf{0} ; \mathbf{0}$ & B & 0;0 & 0 \\
\hline & $e$ & D & 0 & 2 & 0 & 0 & $\mathbf{0} ; 0$ & $\mathbf{0} ; \mathbf{0}$ & 2 & 0 & o \\
\hline
\end{tabular}

The results for the salivary glands females fed for 2, 4 and 6 days from the CG were presented in a previously study [16].

BB: bromophenol; PAS: PAS; B: Baker; AP: phosphatase; VK: von Kossa; *: inactive cell; $\phi$ : not observed cell; the intensity of staining varied from 0-4, being: $\mathbf{0}$ : negative and $\mathbf{4}$ : strongly positive.

Table 4. Protein staining for acini II and III of salivary glands of R. sanguineus females from groups CG and TG fed for 2, 4 and 6 days.

\begin{tabular}{|c|c|c|c|c|c|c|c|}
\hline \multirow{3}{*}{ Acini } & \multirow{3}{*}{ Cells } & \multicolumn{6}{|c|}{ Study Groups } \\
\hline & & \multicolumn{3}{|c|}{ GC } & \multicolumn{3}{|c|}{ GT } \\
\hline & & 2 days of feeding & 4 days of feeding & 6 days of feeding & 2 days of feeding & 4 days of feeding & 6 days of feeding \\
\hline \multirow{6}{*}{ II } & $a$ & 2; 3 & $\mathbf{0} ; 2$ & $\mathbf{0} ; 2$ & 2; 3 & 2 & 2; 3 \\
\hline & $c 1$ & 3 & 2 & 2 & $*$ & 2 & 3 \\
\hline & $c 2$ & ( & ( & ( & ( & ( & ( \\
\hline & $c 3$ & 2 & 2 & 2 & 3 & 2 & 2 \\
\hline & $c 4$ & 0 & 0 & $\phi$ & 0 & $*$ & $\phi$ \\
\hline & $c 5$ & $\mathbf{0} ; 2$ & 2 & $*$ & ( & ( & $*$ \\
\hline \multirow{3}{*}{ III } & $d$ & 3 & 2 & 2; 3 & 3 & 2; 3 & 3 \\
\hline & $e$ & D; 2 & ( & ( & $\mathbf{0} ; 2$ & ( & $\mathbf{0} ; 2$ \\
\hline & $f$ & $\mathbf{0} ; \mathbf{0}$ & - & - & $\mathbf{0} ; 2$ & - & - \\
\hline
\end{tabular}

The results for the salivary glands females fed for 2, 4 and 6 days from the CG were presented in a previously study [16].

*: inactive cell; $\phi$ : not observed; _ _ no secretion granules; the intensity of staining varied from $\mathbf{0}-\mathbf{4}$, being: $\mathbf{0}$ : negative and $\mathbf{4}$ : strongly positive.

Table 5. Polysaccharides staining for acini II and III of salivary glands of R. sanguineus females from groups CG and TG fed for 2, 4 and 6 days.

\begin{tabular}{|c|c|c|c|c|c|c|c|}
\hline \multirow{3}{*}{ Acini } & \multirow{3}{*}{ Cells } & \multicolumn{6}{|c|}{ Study Groups } \\
\hline & & \multicolumn{3}{|c|}{ CG } & \multicolumn{3}{|c|}{ TG } \\
\hline & & 2 days of feeding & 4 days of feeding & 6 days of feeding & 2 days of feeding & 4 days of feeding & 6 days of feeding \\
\hline \multirow{6}{*}{ II } & $a$ & (1) & 0 & 0 & 0; (1) & 0 & 0; 1 \\
\hline & $c 1$ & 3 & 3 & 3 & $*$ & 3 & 3 \\
\hline & $c 2$ & (1) 2 & 2 & 2 & 2 & (1); 2 & (1); 2 \\
\hline & $c 3$ & 3 & 3 & 3 & 3 & 3 & 3 \\
\hline & $c 4$ & 0 & 0 & 0 & 0 & $*$ & $\phi$ \\
\hline & $c 5$ & 1); 2 & (1) 2 & $*$ & 2; 3 & 2 & $*$ \\
\hline \multirow[b]{2}{*}{ III } & $d$ & 0 & 0 & 0 & 0 & 0 & 0; 1 \\
\hline & $e$ & 0 & 0 & 0 & 0 & 0 & 0; 1 \\
\hline
\end{tabular}

The results for the salivary glands females fed for 2, 4 and 6 days from the CG were presented in a previously study [16]. 
*: inactive cell; $\phi$ : not observed cell; _ : no secretion granules; the intensity of staining varied from $\mathbf{0}-\mathbf{4}$, being: $\mathbf{0}$ : negative and $\mathbf{4}$ : strongly positive.

Table 6. Lipids staining for acini II and III of salivary glands of R. sanguineus females from groups CG and TG fed for 2, 4 and 6 days.

\begin{tabular}{|c|c|c|c|c|c|c|c|}
\hline \multirow{3}{*}{ Acini } & \multirow{3}{*}{ Cells } & \multicolumn{6}{|c|}{ Study Groups } \\
\hline & & \multicolumn{3}{|c|}{ CG } & \multicolumn{3}{|c|}{ TG } \\
\hline & & 2 days of feeding & 4 days of feeding & 6 days of feeding & 2 days of feeding & 4 days of feeding & 6 days of feeding \\
\hline \multirow{6}{*}{ II } & $a$ & 3 & 3 & 3 & 2; 3 & 2 & $\mathbf{0} ; 2$ \\
\hline & $c 1$ & 3 & $\phi$ & 3 & $*$ & $\phi$ & $\phi$ \\
\hline & $c 2$ & ( & $\phi$ & 2 & 2 & ( & $\mathbf{0} ; 2$ \\
\hline & $c 3$ & 3 & 3 & 3 & 2 & $\phi$ & 2 \\
\hline & $c 4$ & 0 & ( & 0 & 0 & $*$ & 0 \\
\hline & $c 5$ & 2; 3 & $\phi$ & $*$ & 2; 3 & 3 & $*$ \\
\hline \multirow{3}{*}{ III } & $d$ & 3 & 3 & 3 & $\mathbf{0} ; 3$ & 3 & 3 \\
\hline & $e$ & 2 & 2 & 2 & ( & $\mathbf{0} ; 2$ & 2 \\
\hline & $f$ & 2; 3 & - & - & 2; 3 & - & - \\
\hline
\end{tabular}

The results for the salivary glands females fed for 2, 4 and 6 days from the CG were presented in a previously study [16].

*: inactive cell; $\phi$ : cell not observed; _ : no secretion granules; the intensity of staining varied from $\mathbf{0}-\mathbf{4}$, being: $\mathbf{0}$ : negative and $\mathbf{4}$ : strongly positive.

Table 7. Acid phosphatase staining for acini II and III of salivary glands of R. sanguineus females from groups CG and TG fed for 2, 4 and 6 days.

\begin{tabular}{|c|c|c|c|c|c|c|c|}
\hline \multirow{3}{*}{ Acini } & \multirow{3}{*}{ Cells } & \multicolumn{6}{|c|}{ Study Groups } \\
\hline & & \multicolumn{3}{|c|}{ CG } & \multicolumn{3}{|c|}{ TG } \\
\hline & & 2 days of feeding & 4 days of feeding & 6 days of feeding & 2 days of feeding & 4 days of feeding & 6 days of feeding \\
\hline \multirow{6}{*}{ II } & $a$ & 0 & 0 & 0 & 0 & 0 & 0 \\
\hline & $c 1$ & $\phi$ & 0 & 2 & * & D; 2 & 0 \\
\hline & $c 2$ & 2 & 2 & ( & $\mathbf{0} ; 2$ & D; 2 & $\mathbf{0} ; \mathbf{0}$ \\
\hline & $c 3$ & 2 & 0 & 0 & 2 & ( & o \\
\hline & $c 4$ & 2 & $\mathbf{0} ; \mathbf{0}$ & 0 & o & $*$ & o \\
\hline & $c 5$ & 2 & 2 & * & 2 & D; 2 & * \\
\hline \multirow{3}{*}{ III } & $d$ & 0 & 0 & 0 & O; $\mathbf{0}$ & O; $\mathbf{0}$ & 0; $\mathbf{0}$ \\
\hline & $e$ & 0 & 0 & 0 & 0 & 0 & 0 \\
\hline & $f$ & - ; 2 & - & $\ldots$ & $\mathbf{1} ; \mathbf{2} ; \mathbf{3}$ & 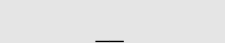 & - \\
\hline
\end{tabular}

The results for the salivary glands females fed for 2, 4 and 6 days from the $\mathbf{C G}$ were presented in a previously study [16].

*: inactive cell; $\phi$ : cell not observed; _ _ no secretion granules; the intensity of staining varied from $\mathbf{0}-\mathbf{4}$, being: $\mathbf{0}$ : negative and $\mathbf{4}$ : strongly positive.

Table 8. Calcium staining for acini II and III of salivary glands of R. sanguineus females from groups CG and TG fed for 2, 4 and 6 days.

\begin{tabular}{|c|c|c|c|c|c|c|c|}
\hline \multirow{3}{*}{ Acini } & \multirow{3}{*}{ Cells } & \multicolumn{6}{|c|}{ Study Groups } \\
\hline & & \multicolumn{3}{|c|}{ CG } & \multicolumn{3}{|c|}{ TG } \\
\hline & & 2 days of feeding & 2 days of feeding & 2 days of feeding & 2 days of feeding & 2 days of feeding & 2 days of feeding \\
\hline \multirow{5}{*}{ II } & $a$ & $\mathbf{0}$ & 0 & 0 & 0 & 0 & 0 \\
\hline & $c 1$ & 3 & 3 & 0 & $*$ & 3 & 3 \\
\hline & $c 2$ & 0 & 0 & 0 & 0 & 0 & 0 \\
\hline & $c 3$ & $\mathbf{0} ; 3$ & 0; 3 & 2 & 3 & 3 & 3 \\
\hline & $c 5$ & 0 & 0 & $*$ & 0 & 0 & $*$ \\
\hline \multirow{3}{*}{ III } & $d$ & 0 & 0 & 0 & 0 & 0 & 0 \\
\hline & $e$ & 0 & 0 & 0 & 0 & 0 & 0 \\
\hline & $f$ & 0 & - & - & 3 & - & - \\
\hline
\end{tabular}

The results for the salivary glands females fed for 2, 4 and 6 days from the CG were presented in a previously study [16].

*: inactive cell; _ : no secretion granules; the intensity of staining varied from $\mathbf{0 - 4}$, being: $\mathbf{0}$ : negative and $\mathbf{4}$ : strongly positive. 

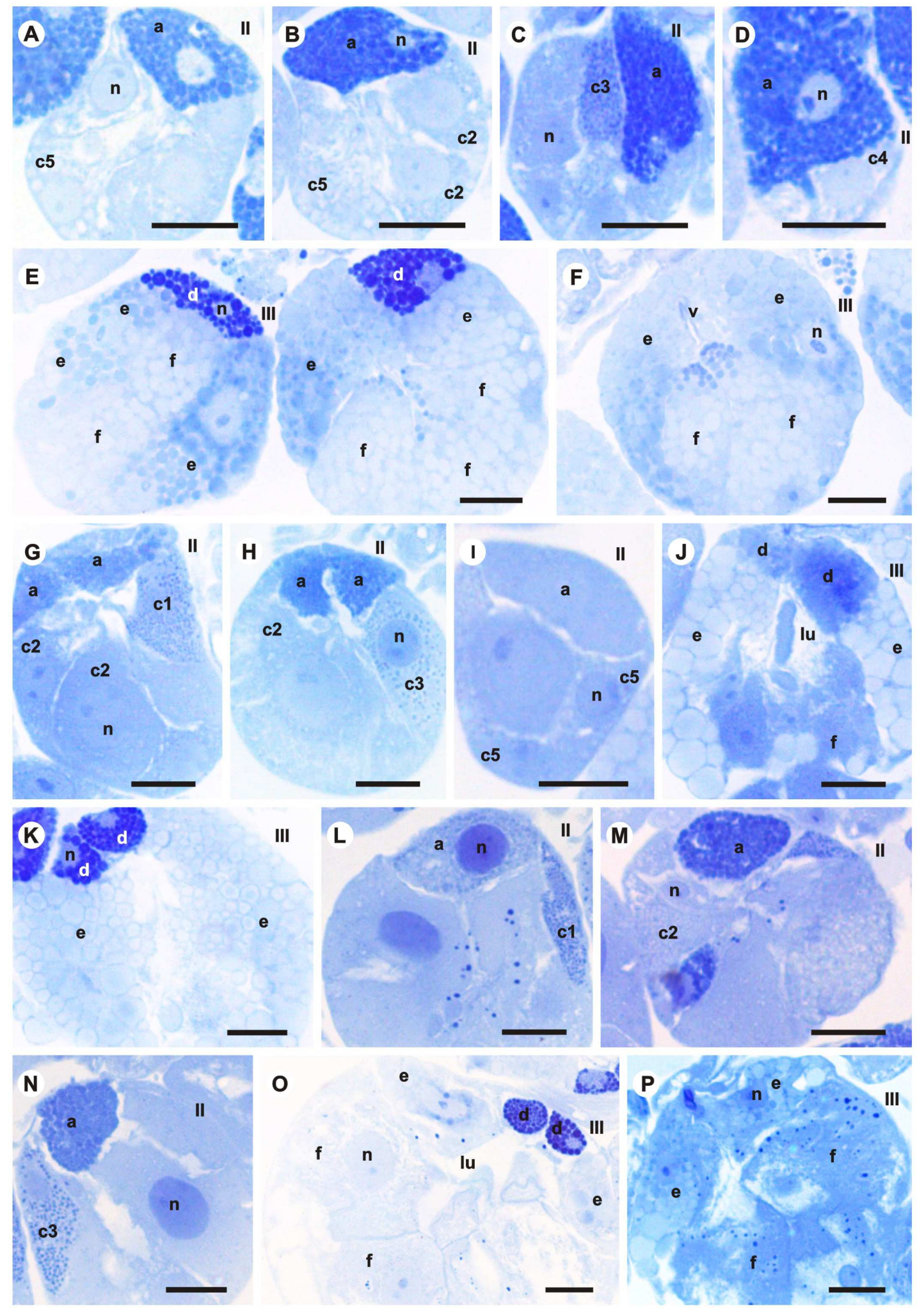

A-F. 2 days of feeding.

G-K. 4 days of feeding.

L-P. 6 days of feeding.

$\mathrm{II}=$ acinus type II; III= acinus type III; $\mathrm{n}=$ nucleus; $\mathrm{lu}=$ acinus lumen; $\mathrm{v}=\mathrm{valve} ; \mathrm{a}=\mathrm{a}$ cell; $\mathrm{c} 1=\mathrm{c} 1 \mathrm{cell} ; \mathrm{c} 2=\mathrm{c} 2 \mathrm{cell} ; \mathrm{c} 3=\mathrm{c} 3 \mathrm{cell} ; \mathrm{c} 4=\mathrm{c} 4 \mathrm{cell} ; \mathrm{c} 5=\mathrm{c} 5$ cell; $\mathrm{d}=\mathrm{d}$ cell; $\mathrm{e}=\mathrm{e}$ cell, $\mathrm{f}=\mathrm{f}$ cell.

Bars: $25 \mu \mathrm{m}$

Figure 1. Histological sections of salivary glands of Rhipicephalus sanguineus females used as test group TG (SGE4). Bromophenol blue technique. 

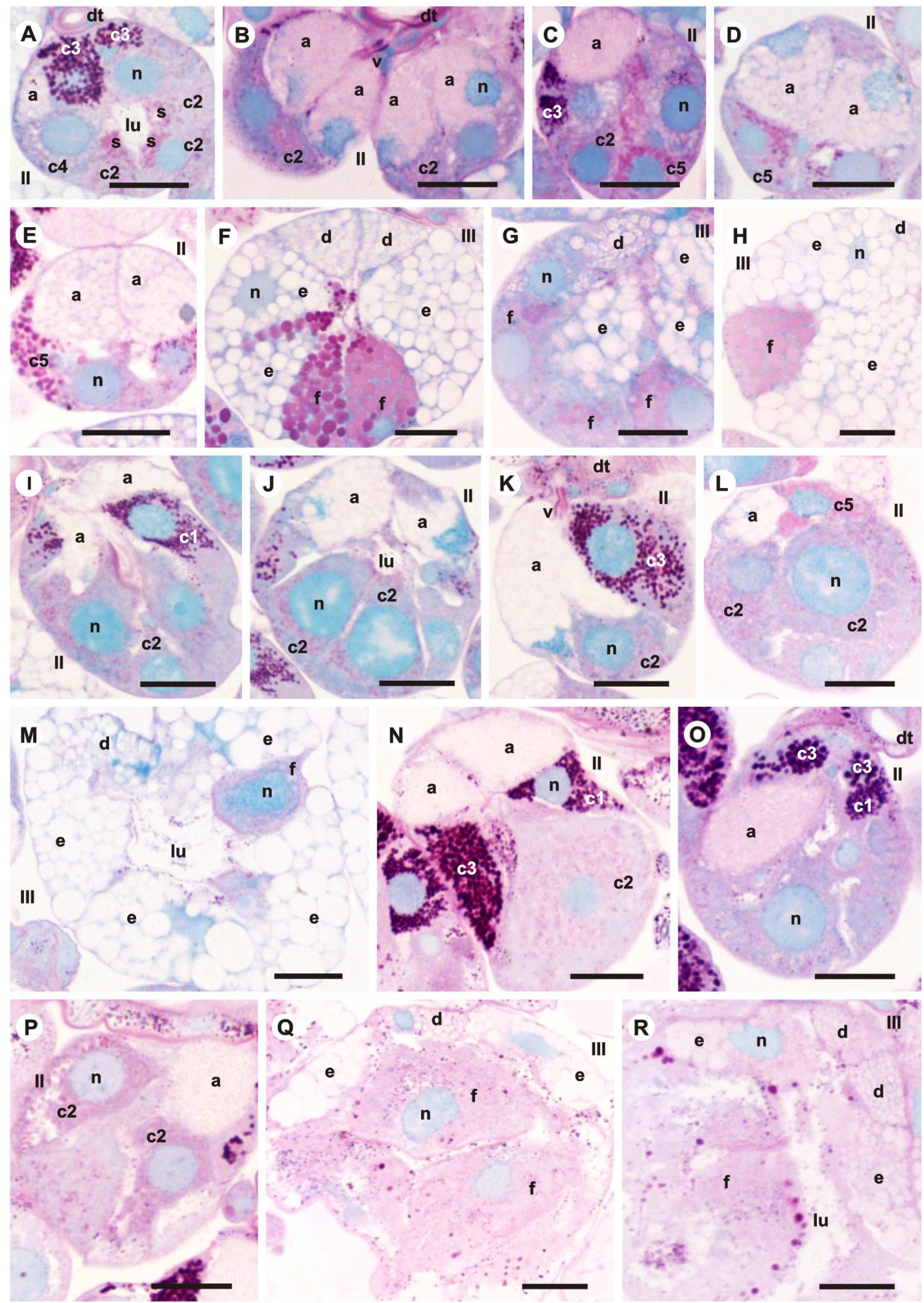

A-H. 2 days of feeding.

I-M. 4 days of feeding.

N-R. 6 days of feeding.

$\mathrm{II}=$ acinus type $\mathrm{II} ; \mathrm{III}=$ acinus type $\mathrm{III} ; \mathrm{n}=$ nucleus; $\mathrm{lu}=$ acinus lumen; $\mathrm{s}=$ secretion granules; $\mathrm{dt}=$ ducto; $\mathrm{v}=\mathrm{valve} ; \mathrm{a}=a$ cell; $\mathrm{c} 1=c 1$ cell; $\mathrm{c} 2=c 2$ cell; $\mathrm{c} 3=c 3$ cell; $\mathrm{c} 4=c 4$ cell; $\mathrm{c} 5=c 5$ cell; $\mathrm{d}=d$ cell; $\mathrm{e}=e$ cell, $\mathrm{f}=f$ cell.

Bars: $25 \mu \mathrm{m}$

Figure 2. Histological sections of salivary glands of Rhipicephalus sanguineus females used as test group TG (SGE4). PAS reaction and counterstained with methyl green. 


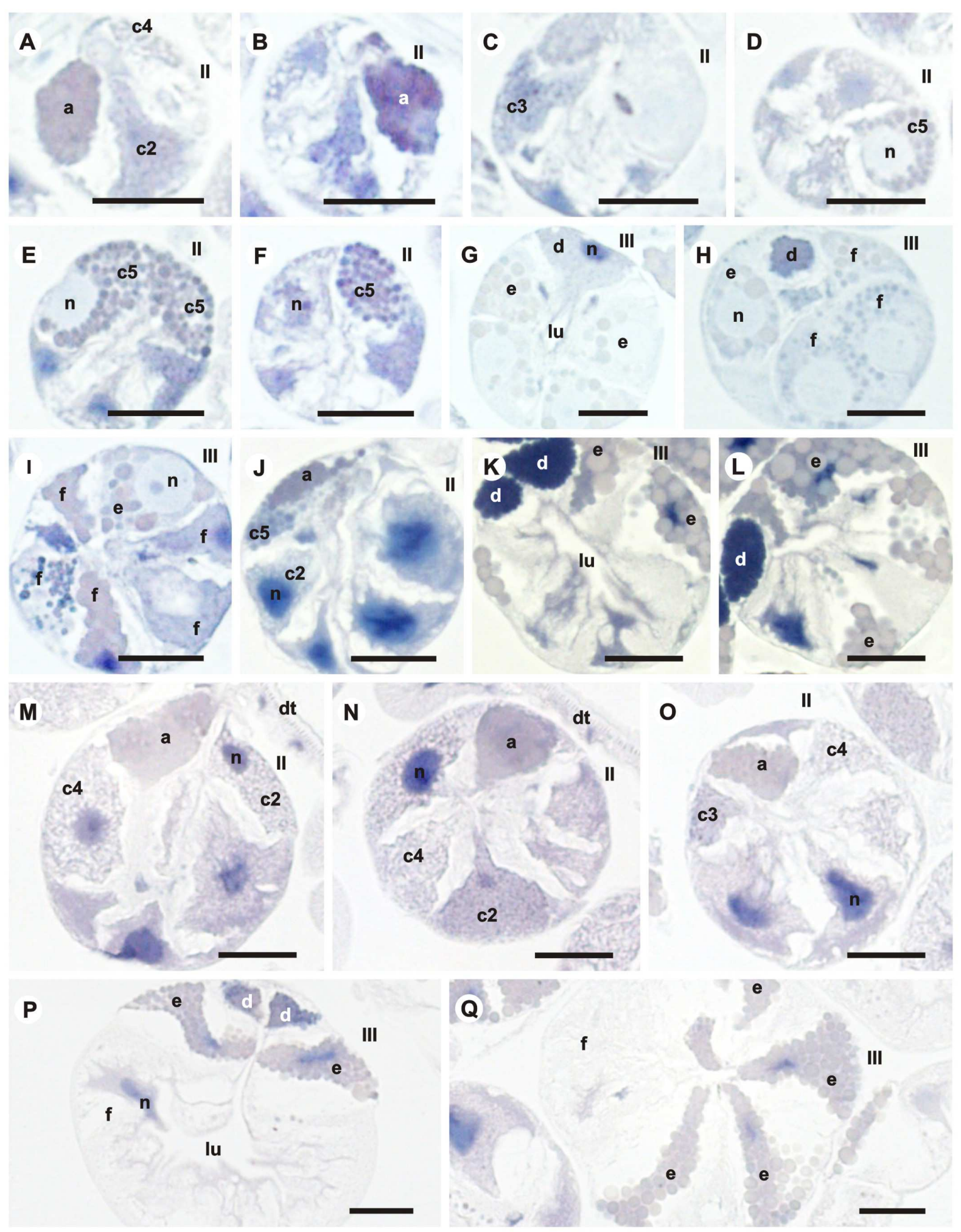

A-I. 2 days of feeding.

J-L. 4 days of feeding.

M-Q. 6 days of feeding.

$\mathrm{II}=$ acinus type II; III = acinus type III; $\mathrm{n}=$ nucleus; $\mathrm{lu}=$ acinus lumen; $\mathrm{a}=a$ cell; $\mathrm{c} 2=c 2$ cell; $\mathrm{c} 3=c 3$ cell; $\mathrm{c} 4=c 4$ cell; $\mathrm{c} 5=c 5$ cell; $\mathrm{d}=d$ cell; $\mathrm{e}=e$ cell, $\mathrm{f}=f$ cell . Bars: $25 \mu \mathrm{m}$

Figure 3. Histological sections of salivary glands of Rhipicephalus sanguineus females used as test group TG (SGE4). Baker technique. 


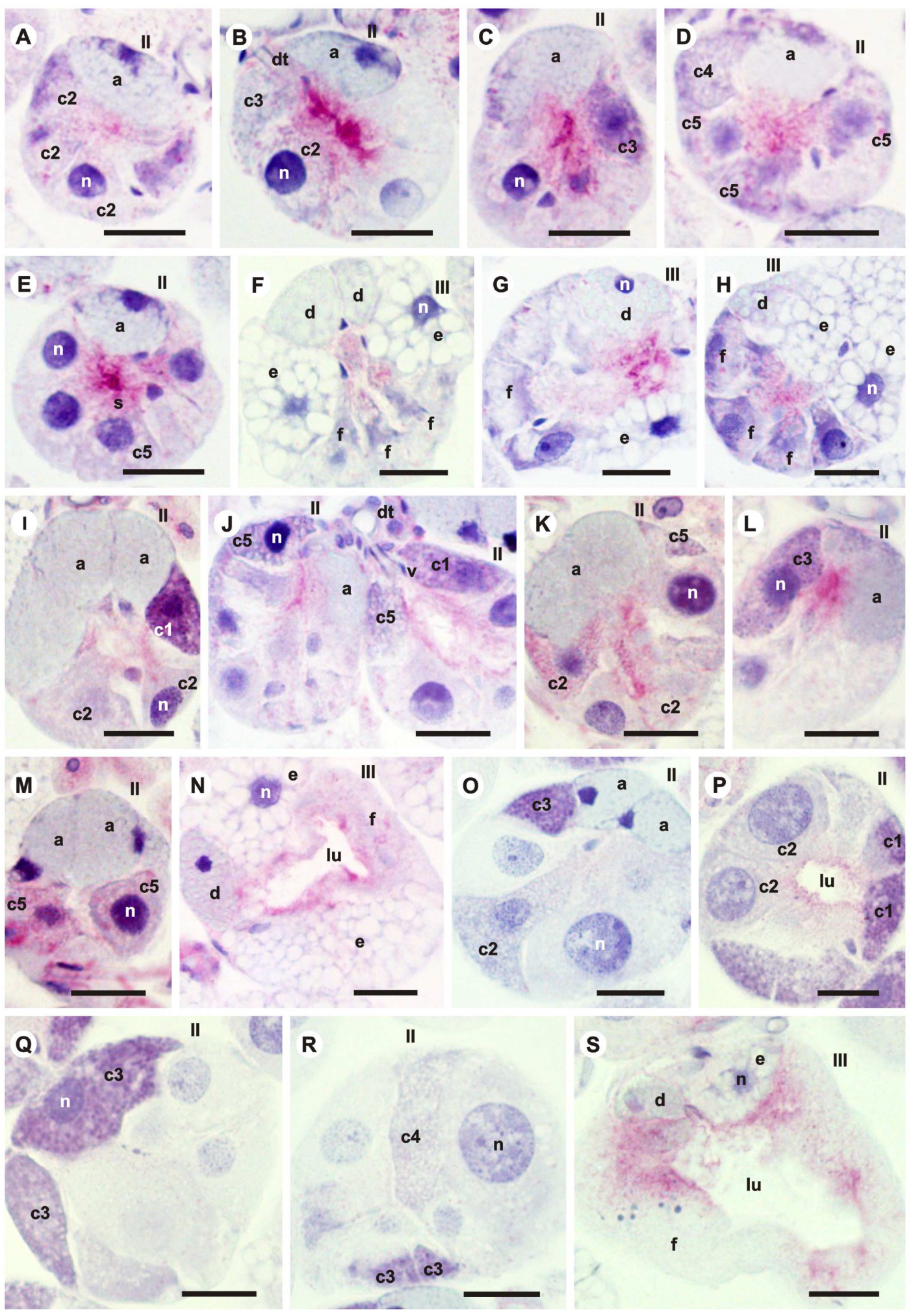

A-H. 2 days of feeding.

I-N. 4 days of feeding.

O-S. 6 days of feeding.

$\mathrm{II}=$ acinus type II; III= acinus type III; $\mathrm{n}=$ nucleus; $\mathrm{dt}=$ ducto; $\mathrm{s}=$ secretion granules; $\mathrm{lu}=$ acinus lumen; $\mathrm{v}=\mathrm{valve} ; \mathrm{a}=a$ cell; $\mathrm{c} 1=c 1 \mathrm{cell} ; \mathrm{c} 2=c 2$ cell; $\mathrm{c} 3=c 3$ cell; $\mathrm{c} 4=c 4$ cell; $\mathrm{c} 5=c 5$ cell; $\mathrm{d}=d$ cell; $\mathrm{e}=e$ cell, $\mathrm{f}=f$ cell.

Bars: $25 \mu \mathrm{m}$

Figure 4. Histological sections of salivary glands of Rhipicephalus sanguineus females used as test group TG (SGE4). Acid phosphatase reaction and counterstained with hematoxylin. 


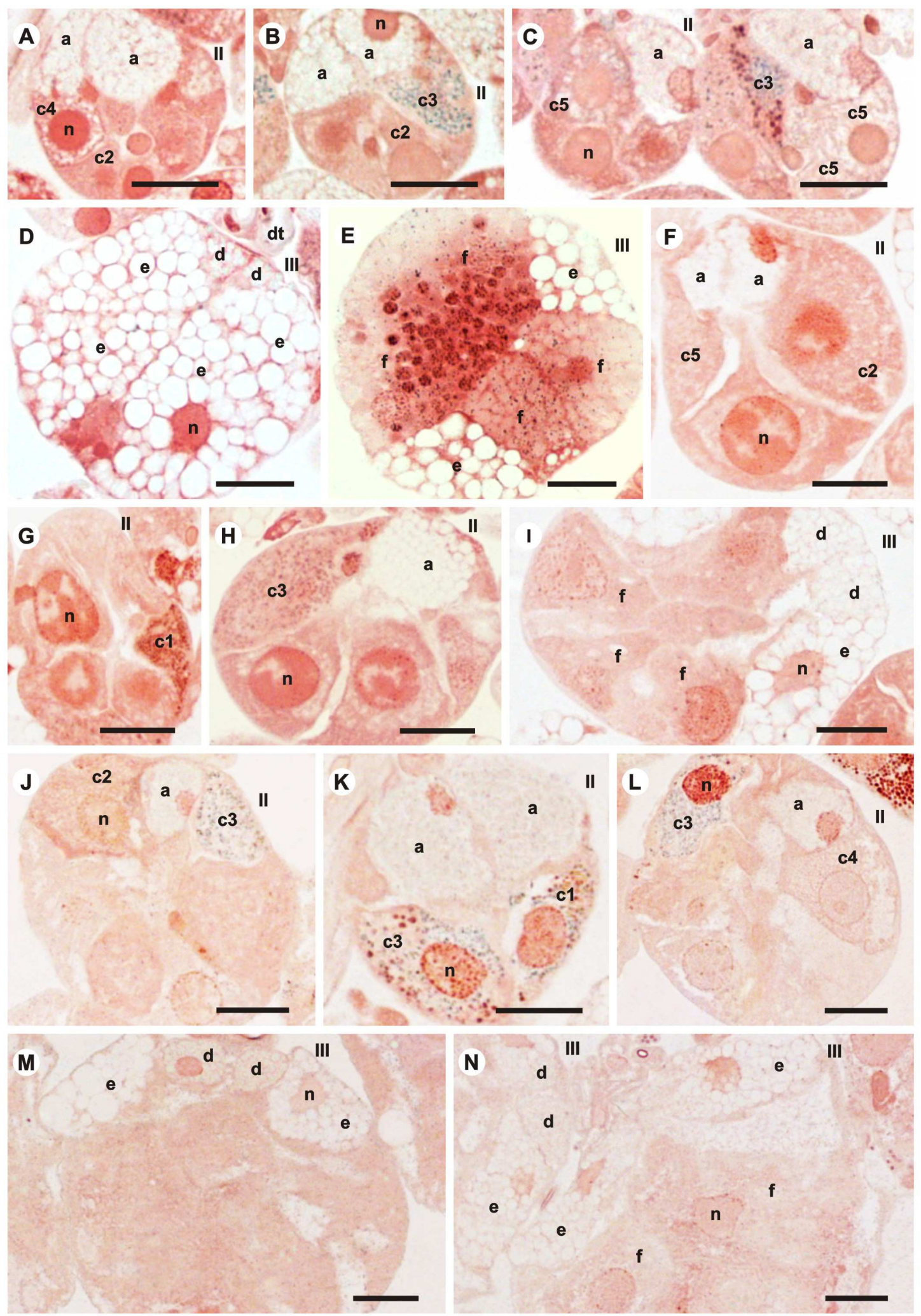

A-E. 2 days of feeding.

F-I. 4 days of feeding.

J-N. 6 days of feeding.

$\mathrm{II}=$ acinus type II; III $=$ acinus type III; $\mathrm{n}=$ nucleus; $\mathrm{dt}=$ ducto; $\mathrm{a}=a$ cell; $\mathrm{c} 1=c 1$ cell; $\mathrm{c} 2=c 2$ cell; $\mathrm{c} 3=c 3$ cell; $\mathrm{c} 4=c 4$ cell; $\mathrm{c} 5=c 5$ cell; $\mathrm{d}=d$ cell; $\mathrm{e}=e$ cell, $\mathrm{f}=f$ cell.

Bars: $25 \mu \mathrm{m}$

Figure 5. Histological sections of salivary glands of Rhipicephalus sanguineus females used as test group TG (SGE4). Von Kossa technique. 


\section{Discussion}

This study showed the alterations which occur in the contents of glycoprotein, lipoprotein, acid phosphatase and calcium in the secretion granules of salivary glands of $R$. sanguineus females fed for 2, 4 and 6 days (test group $=$ TG) in hosts immunized with salivary gland extracts from females fed for 4 days (SGE4). In addition to the histochemical alterations, the loss of activity by $c 1$ (2-day glands) and $c 4$ (4-day glands) secretory cells was also verified, corroborating the reference [9], who demonstrated, through histological evaluation, such alteration after the immunization of the hosts with the same glandular extract used here.

Considering the histochemical alterations here detected in the salivary glands of females from TG group, it can be inferred that the hosts immunized with SGE4 glandular extract developed resistance against the inoculated antigens. In this sense, it can be proposed that the alterations in the salivary glands would be occurring as the organism of the tick tries to compensate the resistance developed by the host, which would permit the continuation and the success of the feeding process, data which corroborate the reference [9].

In this study it was verified that the glandular tissues of females fed for 2 and 6 days were the ones which underwent the most pronounced alterations; however, the alterations in the glands of females fed for 4 days were also significant. This has probably occurred because the glandular extract SGE4 was obtained from salivary glands of females fed for 4 days; i.e., they were in the middle of the glandular cycle [16]; therefore, these organs would contain antigens which would be common both to glandular tissues of females fed for 2 days (beginning of the cycle) and those fed for 6 days (end of glandular cycle). Thus, as suggested by the reference [16], the hosts inoculated with SGE4 extract would present antibodies against the antigens present in the saliva of $R$. sanguineus females in the beginning ( 2 days), middle (4 days) and in the end ( 6 days) of the feeding process, being considered potential to modify the secretory behavior of the salivary glands of the females exposed to the extract, compensating the resistance developed by the host and permitting the continuity and the finalization of the blood feeding process. In this sense, while in CG group the salivary glands of females fed from 2 to 6 days had a gradual reduction in the secreted components, except for calcium and proteins, which remained unaltered in the 4-day glands, in relation to the 2-day and 4-day glands respectively; in TG group it was observed that the 4-day glands, compared with 2-day glands, presented reduced staining for proteins, polysaccharides and acid phosphatase, and the 6-day glands, compared with 4-day glands, had increased proteins and polysaccharides and reduced acid phosphatase. These results suggest that such alterations in TG group would occur due to the resistance developed by the host after the immunization with SGE4 extract. Thus, it can be inferred that the changes observed in the periods of 2 and 4 days would be occurring to modulate the immune-inflammatory system of the host, for this phase antecedes the high consumption of blood by the ectoparasite. On the other hand, the alterations detected in the feeding periods of 4 and 6 days possibly occurred so that the ectoparasite could overcome/contain the hemostatic system of the host, which would make the blood feeding process successful, once this period corresponds to the phase of high and fast blood consumption.

As for the alterations observed specifically in each period of the glandular cycle (2, 4 or 6 days), it was overall observed that, in the 2-day glands, there was an increase in the staining of proteins and calcium, reduction in the staining of lipids and staining of polysaccharides and acid phosphatase remained unaltered. In 4-day glands there was an increase in the staining of proteins, acid phosphatase and calcium; reduction in staining of lipids and the staining of polysaccharides remained unaltered. In 6-day glands an increase in the staining of proteins, polysaccharides and calcium was observed; as well as a reduction in the staining of lipids and acid phosphatase, data that confirm that the glands of females fed for 4 and 6 days presented an increase in staining of different components. A possible explanation for the histochemical alterations found here would be based on the type of immune response developed by the hosts after immunization with SGE4 extract; as the salivary glands, while modifying their secretory behavior, produce a different saliva in order to overcome this resistance, which corroborates the hypothesis by this reference [22], who stated that the pharmacology of the ticks' saliva could be molded according to the host's specific hemostatic defenses.

Thus, in the parasite-immunized host relationship, it would be necessary for the females fed for 2 days to produce a saliva with a higher content of proteins and calcium and less lipids than what is commonly observed in normal situations. This would ensure the efficient modulation of the immuneinflammatory and hemostatic systems of the host, permitting fixation and blood consumption, once such ectoparasites would be in the phase anteceding the period of fast engorgement. On the other hand, the females fed for 4 and 6 days would have to synthetize the saliva with higher protein/acid phosphatase/calcium and proteins/polysaccharides/calcium contents respectively, which would allow the efficient modulation of the host's hemostatic system, allowing the engorgement completion, once in this period the ticks consume larger amounts of blood in less time.

Acini I were not considered in the analysis of salivary glands of this study once they are agranular and do not have a secretory function, acting exclusively on the hydric balance of the ectoparasite [23-24].

In addition, it was also verified in this study that some glandular cells presented more histochemical alterations than others, once the extract used to immunize the hosts would have molecules with different immunogenic capabilities. It is known that the higher the immune response to a particular antigen, the larger the number of alterations in this same 
component of the glands of ticks fed on immunized host; therefore, data here obtained corroborate the reference [14], who reported that not all the components present in the ticks' salivary gland would have antigenic capability.

In this sense, in decrescent order of histochemical alterations, the most affected cells were: $f ; c 2, c 3, c 5 ; a, d$ and $c 4, e$ in the females fed for 2 days, $c 5 ; a, c 2, c 3, d$ and $c 1, e$ in the females fed for 4 days and $a, c 1, c 2, d$ e $c 3, e$ in the females fed for 6 days. According to literature $a$ cells would be responsible for the synthesis of lipoproteins, elements which compose the cement, substance responsible for the formation of the cement cone [3]. However, these cells would contain proteins which would act both in the modulation and formation and maintenance of the feeding lesion [25]. Still according to reference [26], there would be antigenic and non-antigenic proteins in the composition of the cement. The secretions synthesized by $c$ and $f$ cells would be involved in the modulation of the host's local hemostatic reactions [3-4, 6]. In addition, the glycoproteins of $c$ cells would act as anticoagulants [6].

As for the cellular types which were the most affected by the immunologic state of the hosts here studied, it can be inferred that the secretions of $a, c 2, c 3, c 5$ and $d$ cells would present high immunogenic capability and consequent importance for the modulation of the host. However, for $f$ cells, a possible explanation would be in the importance of their secretion for an initial modulation of the immuneinflammatory and hemostatic systems of the host, and not their immunogenicity, a proposal based on the fact that the hosts were not immunized with antigens from $f$ cells; therefore, would not produce antigens, as SGE4 extract did not contain secretion from these cells, active only in the glands of females fed for 2 and 3 days and SGE4 extract was obtained from the glandular tissue of females fed for 4 days.

It was also verified that, among the components which are present in the secretion of salivary glands here analyzed, the ones which presented the most alterations over the glandular cycle were the lipid/acid phosphatase, followed by proteins/polysaccharides and finally calcium, showing the importance of the relation quality/quantity of each bioactive in the modulation of the immune-inflammatory response of the host.

According to literature, the lipidic elements present in the saliva would be in the form of prostaglandins [1, 27-28] purine nucleoside adenosine [28] and prostacyclins [29], molecules which would be part of the sophisticated pharmacological arsenal for the modulation of the local hemostatic reactions of the host, which actuation would interfere in the ectoparasite's capability to remain attached and consuming blood [30].

Prostaglandin and purine nucleoside adenosine were specifically found in the saliva of $R$. sanguineus, and would act in the immune-inflammatory system of the host, ensuring the success of the blood feeding process [28]. According to this reference [31], a lipoprotein of the cement in the secretion granules of $e$ cells in $R$. appendiculatus would have been recognized, having high immunogenic capability, which could explain the greatest number of modifications in lipid staining observed for $R$. sanguineus.

Thus, this study suggested that the glandular extract of $R$. sanguineus females fed for 4 days (SGE4) used for the immunization of the hosts stimulated the development of a kind of immune response which led to significant modifications on the secretory behavior of the salivary glands of the ticks fed on them, demonstrating that the extract would be a reservatory of protecting antigens, important against the infestation by $R$. sanguineus or even other species of ticks, deserving to have detailed studies on the parasite-host relationship from molecular, pharmacologic and immunologic points of view.

\section{Acknowledgments}

This research has been supported by FAPESP (Fundação de Amparo à Pesquisa do Estado de São Paulo) (Grants no. 08/58443-7 and 07/59020-0) and CNPq (Conselho Nacional de Desenvolvimento Científico e Tecnológico) (Grant no. 308733/2006-1 and M.I. Camargo-Mathias and G.H. Bechara academic carrier research fellowships). Part of this work has been facilitated through the Integrated Consortium on Ticks and Tick-borne Diseases (ICTTD-3) supported by the European Union under contract number 510561-INCO.

\section{References}

[1] N. A. Steen, S. C. Barker, P. F. Alewood, "Proteins in the saliva of the Ixodida (ticks): Pharmacological features and biological significance", Toxicon, vol. 47, pp. 1-20, Janery 2006.

[2] B. J. Mans, A. W. H. Neitz, "Adaptation of ticks to a blood feeding environment: Evolution from a functional perspective", Insect Biochem. Mol. Biol., vol. 34, pp. 1-17, Janery 2004.

[3] K. C. Binnington, "Sequential changes in salivary gland structure during attachment and feeding of the cattle tick Boophilus microplus", Int. J. Parasitol., vol. 8, pp. 97-115, April 1978.

[4] A. Walker, J. D. Fletcher, H. S. Gill, "Structural and histochemical changes in the salivary glands of Rhipicephalus appendiculatus during feeding", Int. J. Parasitol. vol. 15, pp. 81-100, February 1985.

[5] H. S. Gill, A. R. Walker, "The salivary glands of Hyalomma anatolicum anatolicum: Nature of salivary gland components and their role in tick attachment and feeding", Int. J. Parasitol., vol. 18, pp. 83-93, February 1988.

[6] D. E. Sonenshine, Biology of ticks. New York: Oxford University Press, 1991.

[7] K. C. S. Furquim, M. I. Camargo-Mathias, L. M. G. F. Hebling, G. C. Roma, G. H. Bechara, "Ticks' response to feeding on host immunized with glandular extracts of Rhipicephalus sanguineus females fed for 2, 4, and 6 days. I. Inactivity or early degeneration of salivary glands?", Parasitol. Res., vol. 109 , pp. 147-162, July 2011 
[8] M. I. Camargo-Matias, K. C. S. Furquim, "The histology as a tool for the understanding of the morphophysiology of the brown dog tick (Rhipicephalus sanguineus)", in Advances in zoology research, vol. 5, O. P. Jenkins, Eds. New York: Nova Science Publishers, 2013, pp. 167-191.

[9] M. I. Camargo-Mathias, K. C. S. Furquim, P. H. Nunes, "Immunomodulatory effects of tick saliva", Invertebrate Surviv. J., vol. 8, pp. 231-240, December 2011.

[10] J. M. C. Ribeiro, G. T. Makoul, J. Levine, D. K. Robinson, A. Spilman, "Antihaemostatic, antiinflammatory and immunosupressive properties of the saliva of a tick, Ixodes dammini”, J. Exp. Med., vol. 161, pp. 332-344, February 1985.

[11] M. Brossar, S. K. Wikel, "Tick immunobiology”, Parasitology, vol. 129, pp. S161-S176, October 2004.

[12] I. M. B. Francicchetti, T. N. Mather, J. N. C. Ribeiro, "Tick saliva is a potent inhibitor of endothelial cell proliferation and angiogenesis", Thromb. Haemost., vol. 94, pp. 167-174, July 2005.

[13] S. Jittapalapong, R. W. Stich, J. C. Gordon, T. E. Wittum, O. O. Barriga, "Performance of female Rhipicephalus sanguineus (Acari: Ixodidae) fed dogs exposed to multiple infestations or immunization with tick salivary gland or midgut tissues", J. Med. Entomol., 37: 601-611, July 2000.

[14] C. M. Wheeler, J. L. Coleman, J. L. Benach, "Salivary gland antigens of Ixodes dammini are glycoproteins that have interspecies cross-reactivity", J. Parasitol., vol. 77, pp. 965973, December 1991.

[15] G. H. Bechara, M. P. J. Szabó, B. R. Ferreira, M. V. Garcia, "Rhipicephalus sanguineus tick in Brazil: feeding and reproductive aspects under laboratorial conditions", Brazil. J. Vet. Parasitol., vol. 4, pp. 61-66, August 1995.

[16] K. C. S. Furquim, M. I. Camargo-Mathias, L. M. G. F. Hebling, G. C. Roma, G. H. Bechara, "Alterations in the secretory behavior of salivary glands of Rhipicephalus sanguineus females (Latreille, 1806) (Acari: Ixodidae) feeding in resistant rabbit", Air Water Borne Diseases, vol. 2, pp. 114, doi: 10.4172/2167-7719.1000114, October 2013.

[17] A. G. E. Pearse, Histochemistry. Theoretical and applied, vol. 2: Analytic Technology, $4^{\text {th }}$ ed., Edinburgh: Churchill Livingston, 1985.

[18] J. F. A. McManus, "Histological demonstration of mucin after periodic acid". Nature, vol. 158, pp. 202, August 1946.

[19] L. C. U. Junqueira, L. M. M. S. Junqueira, Técnicas básicas de citologia e histologia, São Paulo: Editora Santos, 1983.
[20] J. R. Baker, "The histochemical regogntion of lipine". Q. J. Microsc. Sci., vol. 87, pp. 441-470, December 1946.

[21] M. A. Hussein, I. D. Bowen, G. H. Lewis, "The histochemical localization of ATPase, cholinesterase and acid phosphatase activity in Culex pipiens (Diptera, Culicidae) larvae using a methacrylate embedding technique", Cell Biol. Int. Rep., vol. 14, pp. 775-781, September 1990.

[22] J. M. C. Ribeiro, "Role of saliva in blood feeding by arthropods", Annu. Rev. Entomol., vol. 32, pp. 463-478, January 1987.

[23] H. L. McMullen, J. R. Sauer, R. L. Burton, "Possible role in uptake of water vapour by ixodid ticks salivary glands", J. Insect Physiol., vol. 22, pp. 1281-1285, April 1976.

[24] D. Rudolph, W. Knülle, "Uptake of water vapour from air: process, site, and mechanism in ticks", in Comparative Physiology: Water, Ions and Fluid Mechanics, K. SchmidtNielsen, L. Bolis and S. H. P. Maddrell, Eds. Cambridge: Cambridge University Press, 1978, pp.84-85.

[25] A. Mulenga, M. Blandon, R. Khumthong, "The molecular basis of the Amblyomma americanum tick attachment phase", Exp. Appl. Acarol., vol. 41, pp. 267-287, April 2007.

[26] D. E. Sonenshine, Biology of Ticks, vol. 2. Oxford: Oxford University Press, 1993.

[27] S. G. Harris, J. Padilla, L. Koumas, D. Ray, R. P. Phipps, "Prostaglandins as modulators of immunity", Trends Immunol., vol. 23, pp. 144-150, March 2002.

[28] J. C. Oliveira, A. Sá-Nunes, I. M. B. Francischetti, V.Carregaro, E. Anatriello, J. S. Silva, I. K. F. Miranda Santos, J. M. C. Ribeiro, B. R. Ferreira, "Deconstructing saliva. Nonprotein molecules with potent immunomodulatory properties", J. Biol. Chem., vol. 286, pp. 10960-10969, April 2011.

[29] J. M. C. Ribeiro, G. Makoul, D. Robinson, "Ixodes dammini: Evidence for salivary prostacyclin secretion”, J. Parasitol., vol. 74, pp. 1068-1069, December 1988.

[30] I. M. B. Francischetti, A. Sá-Nunes, B. J. Mans, I. M. Santos, J. M. C. Ribeiro, "The role of saliva in tick feeding", Front. Biosci., vol. 14, pp. 2051-2088, January 2010.

[31] R. Bishop, B. Lambson, C. Wells, P. Pandit, J. Osaso, C. Nkonge, S. Morzaria, A. Musoke, V. Nene, "A cement protein of the tick Rhipicephalus sanguineus, located in the secretory e cell granules of the type III salivary gland acini, induces strong antibody response in cattle", Int J Parasitol, vol. 32, pp. 833-842, January 2002. 